\title{
Gastric cancer treated by endoscopic submucosal dissection or endoscopic mucosal resection in Japan from 2004 through 2006: JGCA nationwide registry conducted in 2013
}

\author{
Satoshi Tanabe ${ }^{1} \cdot$ Shigeki Hirabayashi $^{2} \cdot$ Ichiro Oda $^{3}$. \\ Hiroyuki Ono $^{4} \cdot$ Atsushi Nashimoto $^{5} \cdot$ Yoh Isobe $^{6} \cdot$ Isao Miyashiro $^{7}$. \\ Shunichi Tsujitani ${ }^{8} \cdot$ Yasuyuki Seto $^{9} \cdot$ Takeo Fukagawa $^{10}$. \\ Souya Nunobe $^{11} \cdot$ Hiroshi Furukawa $^{12} \cdot$ Yasuhiro Kodera $^{13}$. \\ Michio Kaminishi' ${ }^{14}$ Hitoshi Katai ${ }^{10}$
}

Received: 29 August 2016/Accepted: 29 January 2017/Published online: 15 February 2017

(c) The International Gastric Cancer Association and The Japanese Gastric Cancer Association 2017

\begin{abstract}
Background The Japanese Gastric Cancer Association (JGCA) initiated a new nationwide gastric cancer registry in 2008 and reported the treatment outcomes of patients with primary gastric cancer who underwent surgical therapy in 2001 and 2003. However, the outcomes of endoscopic therapy have not been reported yet.

Methods The JGCA conducted a retrospective nationwide registry in 2013 to investigate the short-term and long-term outcomes of endoscopic mucosal resection or endoscopic submucosal dissection in patients with gastric cancer treated from January 2004 through December 2006. This registry used a computerized database with terminology in accordance with the JGCA classification (13th and 14th editions) and the Japanese Gastric Cancer Treatment Guidelines from 2010. Results Accurate data on 12,647 patients were collected from 126 participating hospitals and analyzed. The
\end{abstract}

Satoshi Tanabe

s-tanabe@kitasato-u.ac.jp

1 Research and Development Center for New Medical Frontiers, Kitasato University School of Medicine, 1-15-1 Kitasato, Minami-ku, Sagamihara, Kanagawa 252-0374, Japan

2 Department of Medical Informatics, Niigata University Medical and Dental Hospital, Niigata, Japan

3 Endoscopy Division, National Cancer Center Hospital, Tokyo, Japan

4 Endoscopy Division, Shizuoka Cancer Center, Shizuoka, Japan

5 Department of Surgery, Niigata Cancer Center Hospital, Niigata, Japan

6 Department of Surgery, National Hospital Organization Tokyo Medical Center, Tokyo, Japan treatment procedure was endoscopic submucosal dissection in $81 \%$ of the patients and endoscopic mucosal resection in $19 \%$. En bloc and R0 resections were achieved in $89 \%$ and $79 \%$ of the patients respectively. The total proportion of patients who underwent curative resection was $69.2 \%$; $43.8 \%$ of patients underwent curative resection for absolute indication lesions, and $25.4 \%$ underwent curative resection for expanded indication lesions. Emergency surgery was performed to treat bleeding or perforation in very few patients $(0.3 \%$ and $0.4 \%$ respectively). The 5 -year followup rate after endoscopic resection was $70 \%$. The 5-year overall survival rate was $91.6 \%$ in patients with absolute indications and $90.3 \%$ in patients with expanded indications after curative resection and $86.5 \%$ in patients who underwent noncurative resection. The 5-year diseasespecific survival rates were $99.9 \%, 99.7 \%$, and $98.7 \%$ in patients with absolute indications who underwent curative

7 Department of Surgery, Osaka Medical Center for Cancer and Cardiovascular Diseases, Osaka, Japan

8 Tottori University Hospital Cancer Center, Yonago, Japan

9 Department of Gastrointestinal Surgery, Graduate School of Medicine, University of Tokyo, Tokyo, Japan

10 Gastric Surgery Division, National Cancer Center Hospital, Tokyo, Japan

11 Department of Gastroenterological Surgery, Cancer Institute Ariake Hospital, Tokyo, Japan

12 Department of Surgery, Kinki University Hospital, Osaka, Japan

13 Department of Surgery, Nagoya University School of Medicine, Nagoya, Japan

14 Department of Surgery, Showa General Hospital, Tokyo, Japan 
resection, patients with expanded indications who underwent curative resection, and patients who underwent noncurative resection respectively.

Conclusion Endoscopic resection of gastric cancer resulted in favorable short-term and long-term outcomes nationwide in Japan. Further efforts to increase the follow-up rate are needed.

Keywords Early gastric cancer - Nationwide registry · 5year survival rate $\cdot 5$-year disease-specific survival rate

\section{Introduction}

The Japanese Gastric Cancer Association (JGCA) reported the results of a nationwide gastric cancer registry in 2006 [1], 2011 [2], and 2013 [3]. The patients were restricted to those who underwent surgical therapy. Since then, remarkable progress has been made in endoscopic treatment of early gastric cancer, which is now performed in increasing numbers of patients. The conventional procedure of endoscopic mucosal resection (EMR) was used as the basis for the development of a procedure for endoscopic submucosal dissection (ESD) $[4,5]$, and the indications for endoscopic treatment have been expanded [6]. We report the short-term and long-term outcomes of registered patients with gastric cancer who underwent endoscopic treatment from 2004 through 2006.

\section{Materials and methods}

The Registration Committee of the JGCA requested hospitals affiliated with JGCA members to enroll consecutive patients with early gastric cancer who underwent EMR or ESD from January 2004 through December 2006. As shown in Table 1, the numbers of participating hospitals and enrolled patients have increased year by year. One hundred twenty-six hospitals participated, and 12,647 patients were enrolled during the 3 years. The Institutional Review Boards of each participating hospital approved the study protocol. In patients with multiple lesions, the main lesion was registered. The main lesion was defined by tumor depth. If multiple lesions had the same tumor depth,

Table 1 Registered institutions and patients by year

\begin{tabular}{lcc}
\hline & Registered institutions & Registered patients \\
\hline 2004 & 89 & 3579 \\
2005 & 99 & 4213 \\
2006 & 108 & 4855 \\
Total & 126 & 12,647 \\
\hline
\end{tabular}

the main lesion was defined by tumor size. The tumor size was based on the histology results. Curability criteria were in accordance with the gastric cancer treatment guidelines described later. All tumors that were resected in a piecemeal fashion were classified as noncurative resection.

This registry used a computerized database with terminology in accordance with the Japanese Classification of Gastric Carcinoma (13th and 14th editions) and the Japanese Gastric Cancer Treatment Guidelines from 2010.

This nationwide registration program was approved by the Ethics Committee of the JGCA.

\section{Results}

Table 2 shows the characteristics of the patients and lesions. The mean age was 71 years. Men accounted for $76 \%$ of the registered patients. The endoscopic treatment procedure was ESD in 10,259 patients $(81.1 \%)$, EMR in 2355 patients $(18.6 \%)$, and other procedures in 30 patients $(0.2 \%)$. The gastric status was an intact stomach in $96 \%$ of the patients, but some patients had a remnant stomach after gastrectomy or a reconstructed gastric tube after esophagectomy. More than $80 \%$ of the lesions were located in the region from the middle to the lower part of the stomach. The largest proportion of lesions was located along the lesser curvature of the stomach. With regard to the macroscopic type, $50 \%$ of the lesions were depressed type, $40 \%$ were elevated type, and $9 \%$ were mixed type. Histologically, $95 \%$ of the lesions were differentiated-type cancer. The median tumor diameter was $15 \mathrm{~mm}$ and the tumor diameter ranged from 0.5 to $169 \mathrm{~mm}$. The depth of tumor invasion extended to the mucosa in $84.1 \%$ of the patients, and was less than $500 \mu \mathrm{m}$ from the muscularis mucosae in $7.9 \%$ of the patients and $500 \mu \mathrm{m}$ or more from the muscularis mucosae in $7.2 \%$ of the patients. With regard to lymphovascular invasion, $4.5 \%$ of the patients had lymphatic invasion and $2.3 \%$ had venous invasion. Ulcers (including open ulcers and ulcer scars) were found in $10.5 \%$ of the patients.

\section{Short-term outcomes of endoscopic resection}

With regard to short-term outcomes (Table 3), the en bloc resection rate was $89.3 \%$. Horizontal margins were negative in $81.6 \%$ of patients, and vertical margins were negative in $94.5 \%$. The rate of en bloc resection with negative margins was $78.9 \%$. The total proportion of patients who underwent curative resection was $69.2 \%$; $43.8 \%$ of patients underwent curative resection for absolute indication lesions, and $25.4 \%$ underwent curative resection for expanded indication lesions. Noncurative resection was performed in 3704 patients $(29.3 \%)$. Of the patients who 
Table 2 Patient $(n=12,647)$ and lesion characteristics

\begin{tabular}{|c|c|c|}
\hline & Number or value & Percentage \\
\hline \multicolumn{3}{|l|}{ Age (years) } \\
\hline Median $^{\mathrm{a}}$ & $71(18-96), 12,644$ & $>99.9$ \\
\hline Missing data & 3 & $<0.1$ \\
\hline \multicolumn{3}{|l|}{ Sex } \\
\hline Male & 9585 & 75.8 \\
\hline Female & 3062 & 24.2 \\
\hline \multicolumn{3}{|c|}{ Method of endoscopic resection } \\
\hline ESD & 10,259 & 81.1 \\
\hline EMR & 2355 & 18.6 \\
\hline Other & 30 & 0.2 \\
\hline Missing data & 3 & $<0.1$ \\
\hline \multicolumn{3}{|l|}{ Treatment history } \\
\hline Primary treatment & 12,298 & 97.2 \\
\hline Posttreatment & 349 & 2.8 \\
\hline \multicolumn{3}{|l|}{ Gastric status } \\
\hline Intact stomach & 12,196 & 96.4 \\
\hline $\begin{array}{l}\text { Remnant stomach after } \\
\text { gastrectomy }\end{array}$ & 356 & 2.8 \\
\hline $\begin{array}{c}\text { Gastric tube after } \\
\text { esophagectomy }\end{array}$ & 92 & 0.7 \\
\hline Missing data & 3 & $<0.1$ \\
\hline \multicolumn{3}{|l|}{ Tumor location } \\
\hline \multicolumn{3}{|l|}{ Three gastric regions } \\
\hline Upper & 2251 & 17.8 \\
\hline Middle & 4889 & 38.7 \\
\hline Lower & 5502 & 43.5 \\
\hline Missing data & 5 & $<0.1$ \\
\hline \multicolumn{3}{|c|}{ Four parts of the gastric circumference } \\
\hline Lesser curvature & 5346 & 42.3 \\
\hline Greater curvature & 2268 & 17.9 \\
\hline Anterior wall & 2327 & 18.4 \\
\hline Posterior wall & 2702 & 21.4 \\
\hline Missing data & 4 & $<0.1$ \\
\hline \multicolumn{3}{|l|}{ Macroscopic type } \\
\hline Depressed & 6378 & 50.4 \\
\hline Elevated & 5014 & 39.6 \\
\hline Mixed & 1157 & 9.1 \\
\hline Undetermined & 93 & 0.7 \\
\hline Missing data & 5 & $<0.1$ \\
\hline \multicolumn{3}{|l|}{ Histological type } \\
\hline pap & 428 & 3.4 \\
\hline tub1 & 10,048 & 79.4 \\
\hline tub2 & 1648 & 13.0 \\
\hline por & 198 & 1.6 \\
\hline sig & 234 & 1.9 \\
\hline muc & 10 & 0.1 \\
\hline Other & 52 & 0.4 \\
\hline Undetermined & 29 & 0.2 \\
\hline Tumor size (mm) & & \\
\hline
\end{tabular}

Table 2 continued

\begin{tabular}{|c|c|c|}
\hline & Number or value & Percentage \\
\hline Median $^{\mathrm{a}}$ & $\begin{array}{l}15.0(0.5-169.0) \\
12,424\end{array}$ & 98.2 \\
\hline Missing data & 223 & 1.8 \\
\hline \multicolumn{3}{|l|}{ Depth of invasion } \\
\hline M & 10,639 & 84.1 \\
\hline SM1 & 1005 & 7.9 \\
\hline Deeper than or equal to SM2 & 914 & 7.2 \\
\hline Other & 15 & 0.1 \\
\hline Undetermined & 70 & 0.6 \\
\hline Missing data & 4 & $<0.1$ \\
\hline \multicolumn{3}{|l|}{ Lymphatic invasion } \\
\hline Negative & 11,956 & 94.5 \\
\hline Positive & 566 & 4.5 \\
\hline Undetermined & 119 & 0.9 \\
\hline Missing data & 6 & $<0.1$ \\
\hline \multicolumn{3}{|l|}{ Venous invasion } \\
\hline Negative & 12,234 & 96.7 \\
\hline Positive & 287 & 2.3 \\
\hline Undetermined & 120 & 0.9 \\
\hline Missing data & 6 & $<0.1$ \\
\hline \multicolumn{3}{|l|}{ Ulcer findings } \\
\hline Absent & 11,084 & 87.6 \\
\hline Present & 1325 & 10.5 \\
\hline Undetermined & 231 & 1.8 \\
\hline Missing data & 7 & 0.1 \\
\hline
\end{tabular}

$E M R$ endoscopic mucosal resection, ESD endoscopic submucosal dissection, $M$ intramucosa, mис mucinous adenocarcinomapap papillary adenocarcinoma, por poorly differentiated adenocarcinoma, $S M 1$ depth of invasion less than $500 \mu \mathrm{m}$ from the muscularis mucosae, SM2 depth of invasion $500 \mu \mathrm{m}$ or more from the muscularis mucosae, tub1 well-differentiated tubular adenocarcinoma, tub2 moderately differentiated tubular adenocarcinoma, sig signet ring cell carcinoma

a The range is given in parentheses

underwent noncurative resection, $63.2 \%$ received no additional treatment, $25.2 \%$ underwent surgical gastrectomy, and 3.2\% underwent additional endoscopic resection. Endoscopic ablation was performed in 155 patients (4.2\%). Emergency surgery was performed to treat bleeding in 38 patients $(0.3 \%)$ and perforation in 45 patients $(0.4 \%)$. The short-term outcomes are shown according to the method of endoscopic treatment in Table 4. The rate of en bloc resection was significantly higher for ESD (94.5\%) than for EMR $(66.8 \% ; p<0.01)$. The rate of complete en bloc resection was significantly higher for ESD (86.0\%) than for EMR $(48.2 \% ; p<0.01)$. The curative resection rate for absolute indication lesions and expanded indication lesions combined was significantly higher for ESD (75.1\%) than for EMR $(43.7 \% ; p<0.01)$. The rate of emergency 
Table 3 Short-term outcomes ( $n=12,647$ patients)

\begin{tabular}{|c|c|c|}
\hline & Number & Percentage \\
\hline \multicolumn{3}{|l|}{ Resection type } \\
\hline En bloc resection & 11,296 & 89.3 \\
\hline Fractional resection & 1347 & 10.7 \\
\hline Resection not possible & 3 & $<0.1$ \\
\hline Missing data & 1 & $<0.1$ \\
\hline \multicolumn{3}{|l|}{ Horizontal margin involvement } \\
\hline Negative & 10,319 & 81.6 \\
\hline Positive & 950 & 7.5 \\
\hline Undetermined & 1365 & 10.8 \\
\hline Missing data & 13 & 0.1 \\
\hline \multicolumn{3}{|l|}{ Vertical margin involvement } \\
\hline Negative & 11,951 & 94.5 \\
\hline Positive & 382 & 3.0 \\
\hline Undetermined & 302 & 2.4 \\
\hline Missing data & 12 & 0.1 \\
\hline \multicolumn{3}{|l|}{ En bloc resection with negative margins } \\
\hline Fulfilled & 9975 & 78.9 \\
\hline Not fulfilled & 2669 & 21.1 \\
\hline Missing data & 3 & $<0.1$ \\
\hline \multicolumn{3}{|l|}{ Curability } \\
\hline Curative resection for absolute indication lesions & 5544 & 43.8 \\
\hline Curative resection for expanded indication lesions & 3210 & 25.4 \\
\hline Noncurative resection & 3704 & 29.3 \\
\hline Undetermined & 189 & 1.5 \\
\hline \multicolumn{3}{|l|}{ Additional treatment for noncurative resection } \\
\hline No treatment & 2342 & 63.2 \\
\hline Surgical gastrectomy & 933 & 25.2 \\
\hline Repeated endoscopic resection & 118 & 3.2 \\
\hline Endoscopic ablation & 155 & 4.2 \\
\hline Other & 89 & 2.4 \\
\hline Missing data & 67 & 1.8 \\
\hline \multicolumn{3}{|l|}{ Emergency operation for bleeding } \\
\hline Operated on & 38 & 0.3 \\
\hline Not operated on & 12,608 & 99.7 \\
\hline Missing data & 1 & $<0.1$ \\
\hline \multicolumn{3}{|l|}{ Emergency operation for perforation } \\
\hline Operated on & 45 & 0.4 \\
\hline Not operated on & 12,602 & 99.6 \\
\hline
\end{tabular}

surgery for complications did not differ significantly between ESD and EMR $(0.3 \%$ vs $0.4 \%$ for bleeding and $0.3 \%$ vs $0.4 \%$ for perforation).

\section{Long-term outcomes of endoscopic resection}

The 5-year follow-up rate after endoscopic resection was $70 \%$. Figure 1 shows the 5-year overall survival rates according to curability. The 5-year overall survival rate was $91.6 \%$ after curative resection of absolute indication lesions and $90.3 \%$ after curative resection of expanded indication lesions, as compared with $86.5 \%$ after noncurative resection, which was slightly lower. Figure 2 shows the disease-specific survival rates according to curability. The 5-year disease-specific survival rate was $99.9 \%$ after curative resection of absolute indication lesions and $99.7 \%$ after curative resection of expanded indication lesions, indicating good outcomes. In patients who underwent noncurative resection, the 5-year disease-specific survival rate was $98.7 \%$, indicating good outcomes. 
Table 4 Short-term outcomes according to the method of endoscopic resection

\begin{tabular}{|c|c|c|c|c|c|}
\hline & \multicolumn{2}{|c|}{$\operatorname{ESD}(n=10,259)$} & \multicolumn{2}{|c|}{$\operatorname{EMR}(n=2355)$} & \multirow[t]{2}{*}{$p$} \\
\hline & Number & Percentage & Number & Percentage & \\
\hline Resection type & & & & & $<0.01$ \\
\hline En bloc resection & 9698 & 94.5 & 1573 & 66.8 & \\
\hline Fractional resection & 557 & 5.4 & 781 & 33.2 & \\
\hline Resection not possible & 2 & $<0.1$ & 0 & 0 & \\
\hline Missing data & 2 & $<0.1$ & 1 & $<0.1$ & \\
\hline Horizontal margin involvement & & & & & $<0.01$ \\
\hline Negative & 9116 & 88.9 & 1181 & 50.1 & \\
\hline Positive & 494 & 4.8 & 451 & 19.2 & \\
\hline Undetermined & 645 & 6.3 & 714 & 30.3 & \\
\hline Missing data & 4 & $<0.1$ & 9 & 0.4 & \\
\hline Vertical margin involvement & & & & & $<0.01$ \\
\hline Negative & 9783 & 95.4 & 2138 & 90.8 & \\
\hline Positive & 298 & 2.9 & 84 & 3.6 & \\
\hline Undetermined & 175 & 1.7 & 124 & 5.3 & \\
\hline Missing data & 3 & $<0.1$ & 9 & 0.4 & \\
\hline En bloc resection with negative margins & & & & & $<0.01$ \\
\hline Fulfilled & 8819 & 86.0 & 1134 & 48.2 & \\
\hline Not fulfilled & 1438 & 14.0 & 1220 & 51.8 & \\
\hline Missing data & 2 & $<0.1$ & 1 & $<0.1$ & \\
\hline Curability & & & & & $<0.01$ \\
\hline Curative resection for absolute indication lesions & 4648 & 45.3 & 879 & 37.3 & \\
\hline Curative resection for expanded indication lesions & 3056 & 29.8 & 150 & 6.4 & \\
\hline Noncurative resection & 2413 & 23.5 & 1279 & 54.3 & \\
\hline Undetermined & 142 & 1.4 & 47 & 2.0 & \\
\hline Additional treatment for noncurative resection & & & & & $<0.01$ \\
\hline No treatment & 1421 & 58.9 & 913 & 71.4 & \\
\hline Surgical gastrectomy & 787 & 32.6 & 145 & 11.3 & \\
\hline Repeated endoscopic resection & 51 & 2.1 & 65 & 5.1 & \\
\hline Endoscopic ablation & 62 & 2.6 & 93 & 7.3 & \\
\hline Other & 32 & 1.3 & 56 & 4.4 & \\
\hline Missing data & 60 & 2.5 & 7 & 0.5 & \\
\hline Emergency operation for bleeding & & & & & 0.226 \\
\hline Operated on & 28 & 0.3 & 10 & 0.4 & \\
\hline Not operated on & 10,231 & 99.7 & 2345 & 99.6 & \\
\hline Missing data & 0 & 0 & 0 & 0 & \\
\hline Emergency operation for perforation & & & & & 0.540 \\
\hline Operated on & 35 & 0.3 & 10 & 0.4 & \\
\hline Not operated on & 10,224 & 99.7 & 2345 & 99.6 & \\
\hline
\end{tabular}

$E M R$ endoscopic mucosal resection, ESD endoscopic submucosal dissection

\section{Discussion}

EMR [7] was developed in the 1980s as an endoscopic treatment for early gastric cancer. The development of EMR made possible the endoscopic resection of small differentiated mucosal carcinomas measuring $2 \mathrm{~cm}$ or less in diameter. However, EMR often had to be performed in a piecemeal fashion because of technical limitations [8], and histopathological evaluation was difficult in an appreciable number of patients. In the late 1990s, ESD was developed to make possible the en bloc resection of larger lesions [4] and rapidly became popular. Gotoda et al. [6] attempted to expand the clinical indications for ESD in accordance with the positioning of ESD in clinical trials. However, the long- 
Fig. 1 Overall survival according to curability

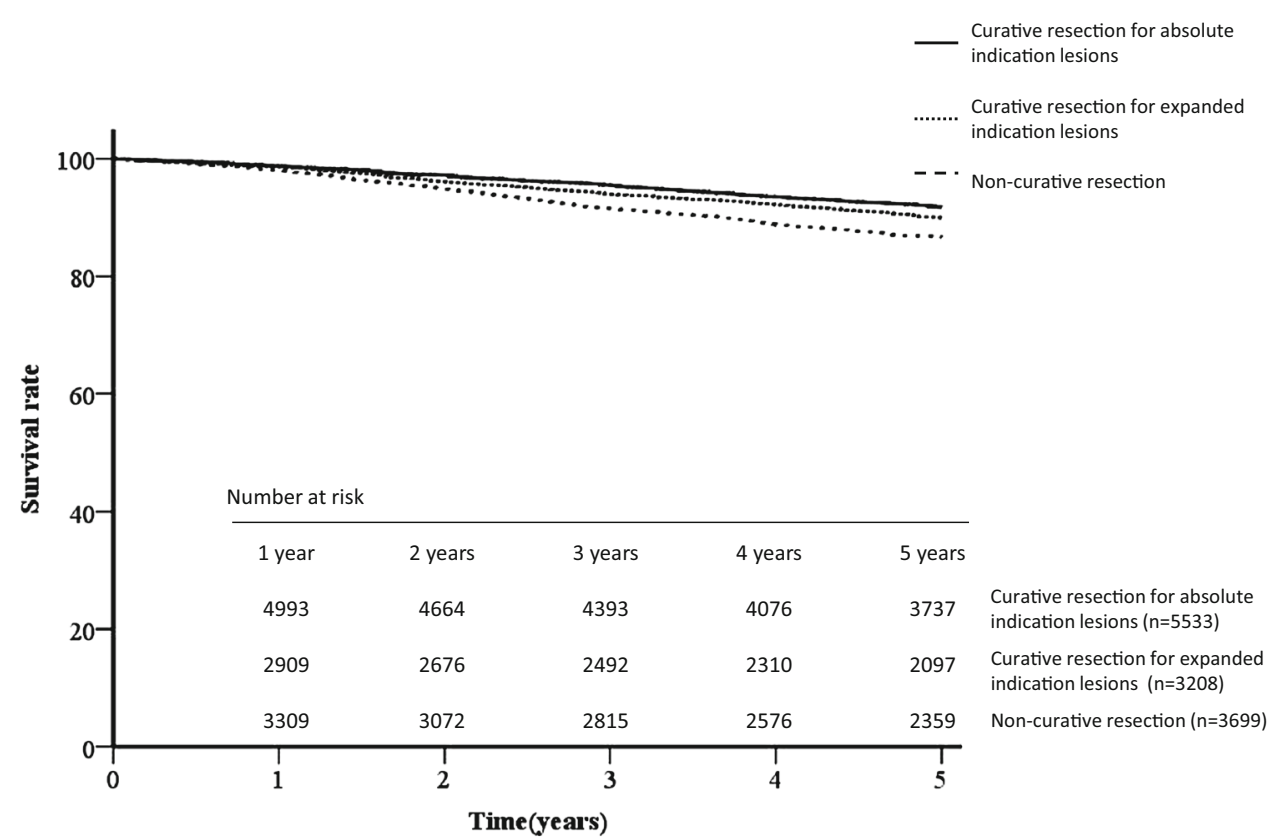

Fig. 2 Disease-specific survival according to curability

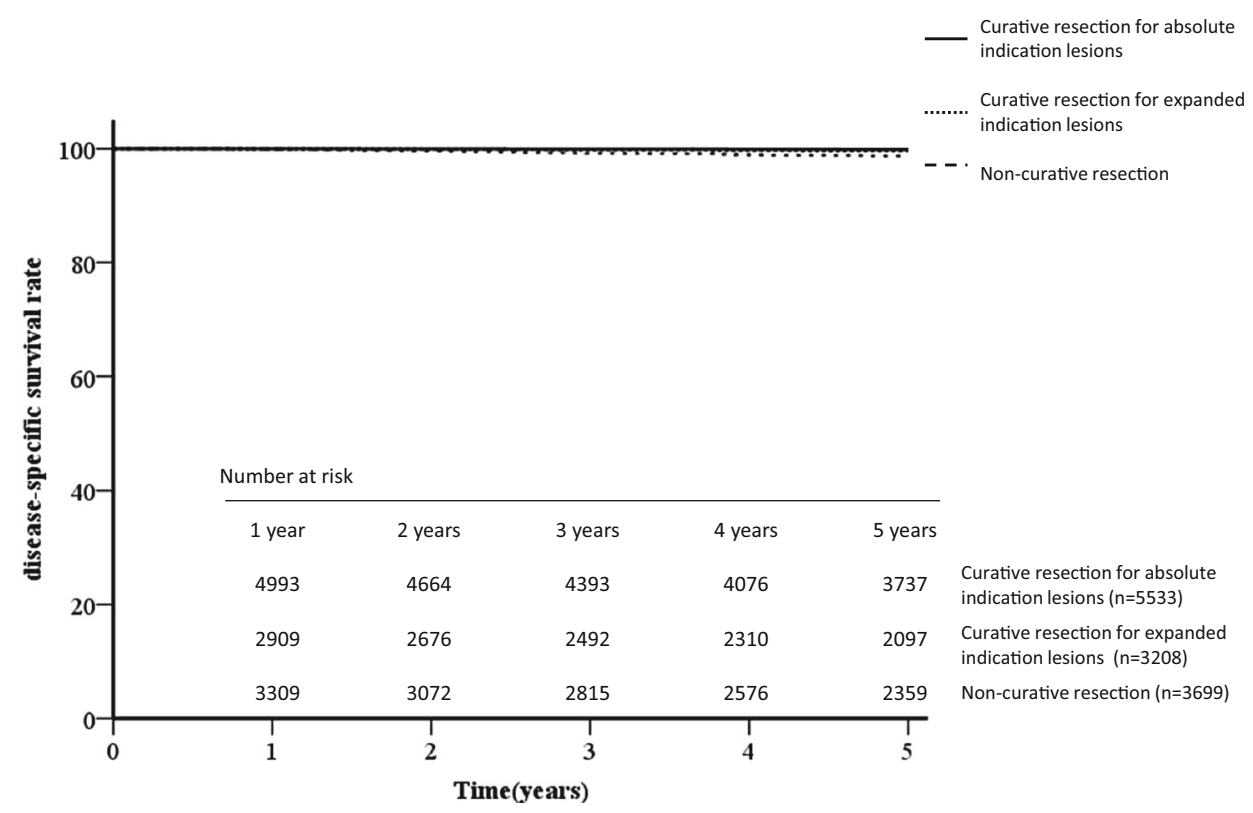

term outcomes of ESD for expanded indication lesions remain unclear. Although ESD has several advantages, such as a high rate of en bloc resection and accurate histopathological assessment, it also has drawbacks when compared with EMR, including a higher incidence of complications such as posttreatment bleeding and perforation [9]; moreover, a longer time is required to master the technique for ESD as compared with that for EMR.

Oda et al. [10] retrospectively studied 714 patients who underwent endoscopic resection of early gastric cancer in 11 Japanese hospitals in 2001. During the study, EMR was performed in more than half of the patients (EMR in 411 patients and ESD in 303 patients). In the present study, we compiled data on patients who underwent endoscopic treatment of early gastric cancer in more than 100 Japanese hospitals from 2004 through 2006. To our knowledge, studies of a similar size have not been reported previously. ESD for treatment of early gastric cancer was approved for coverage by the Japanese National Health Insurance in April 2006 and was soon performed in more than $80 \%$ of patients, indicating its rapid acceptance. ESD is thus considered a very therapeutically useful procedure.

With regard to short-term outcomes, the rates of en bloc resection and complete en bloc resection achieved by ESD 
(95\% and $86 \%$ respectively) were significantly higher than those achieved by EMR ( $67 \%$ and $48 \%$ respectively). The rate of conversion to open surgery because of complications such as bleeding and perforation did not differ between ESD and EMR. The incidences of late bleeding and perforation have been reported to be higher for ESD than for EMR [9], but some studies found no difference in complication rates between ESD and EMR after the procedure for ESD had been technically mastered [11, 12]. In our study, the proportions of patients who had serious complications requiring surgery did not differ significantly between ESD and EMR.

With regard to long-term outcomes according to curability, several studies have reported highly favorable 5-year overall and disease-specific survival rates in patients with early gastric cancer who underwent curative ESD $[13,14]$. In our study, the 5-year disease-specific survival rate was nearly $100 \%$ in patients who underwent curative resection for absolute indication lesions or expanded indication lesions. In studies comparing ESD with surgery, the overall survival rate after ESD was similar to that after surgery, and the benefit of ESD as compared with surgery included fewer late complications and a shorter hospital stay $[15,16]$.

Outcomes in patients with expanded indication lesions have been sporadically reported by various groups of investigators, and many studies showed no difference in outcomes in comparison with patients who had absolute indication lesions $[14,17,18]$. These studies were conducted in relatively small numbers of patients at single centers. In our study, $5(0.16 \%)$ of 3056 patients with expanded indication lesions that were curatively resected by ESD died of gastric cancer. In another multicenter collaborative study that we conducted, $6(0.14 \%)$ of 4202 patients with expanded indication lesions had metastatic recurrence [19]. Prospective studies of expanded indication lesions, including the JCOG0607 study [20], the JCOG1009 study [21], and the J-WEB/EGC study [22], have been conducted, and the results of these studies are awaited.

The results of previous studies suggest that expanded indication lesions might be associated with extremely low risks of metastatic recurrence and death from gastric cancer. Kikuchi et al. [23] reported that $3(0.35 \%)$ of 851 patients with early gastric cancer died after surgical therapy, indicating that surgery is also associated with a certain level of risk. Given this background, endoscopic therapy is considered one of the treatment options for expanded indication lesions provided that informed consent is obtained from patients after they are given an adequate explanation about the risk of metastatic recurrence, albeit the incidence is less than $1 \%$.

This is the first time the results of a nationwide study performed by the JGCA that analyzed the outcomes of endoscopic treatment of early gastric cancer have been reported. We compiled and analyzed data on more than 12,000 patients from 126 Japanese hospitals. Our results indicate that endoscopic treatment had good short-term and long-term outcomes in patients with early gastric cancer. The present study was performed during the shift from EMR to ESD and compared the treatment outcomes of these procedures. We therefore consider our results to be valuable. Future participation of more hospitals in nationwide registration may allow various new findings, including the long-term outcomes of patients with expanded indication lesions, to be obtained. On the other hand, the proportion of patients who could be followed up for at least 5 years was only $70 \%$, which was not satisfactory. We will attempt to increase the follow-up rate in future studies and thereby report more accurate results.

Acknowledgements The Japanese Gastric Cancer Association Registration Committee is indebted to the great efforts of the member hospitals in registering accurate and detailed data for this project.

\section{Compliance with ethical standards}

All procedures followed were in accordance with the ethical standards of the responsible committee on human experimentation (institutional and national) and with the Helsinki Declaration of 1964 and later versions.

Conflict of interest The authors declare that they have no conflict of interest

\section{Appendix: Participating hospitals}

Data on gastric cancer patients in this report were collected from the gastrointestinal or surgical departments of the following 126 hospitals (in alphabetical order): Ageo Central General Hospital, Aichi Cancer Center Hospital, Aichi Medical University, Aizawa Hospital, Ako City Hospital, Cancer Institute Hospital, Center Hospital of National Center for Global Health and Medicine, Chiba University Hospital, Dokkyo Medical University Hospital, Ebina General Hospital, Fukui Prefectural Hospital, Fukui Red Cross Hospital, Fukuiken Saiseikai Hospital, Fukuoka University Chikushi Hospital, Fukushima Medical University Hospital, Fukushima Rosai Hospital, Gunma University Hospital, Hamamatsu University Hospital, Health Insurance Hitoyoshi General Hospital, Higashiyamato Hospital, Himeji Central Hospital, Hirosaki University Hospital, Hiroshima City Asa Hospital, Hiroshima City Hospital, Hiroshima Prefectural Hospital, Hiroshima University Hospital, Hokkaido Cancer Center, Hokkaido University Hospital, Hyogo Cancer Center, Hyogo College of Medicine, Ishikawa Prefectural Central Hospital, Iwate Prefectural Central Hospital, JA Hiroshima 
General Hospital, Jichi Medical University Hospital, Juntendo University Juntendo Hospital, Jusendo General Hospital, Kanazawa University Hospital, Kashiwa Kousei General Hospital, Kawasaki Medical School Hospital, Keiyukai Sapporo Hospital, Kinki Central Hospital, Kitano Hospital, Kitasato University East Hospital, KKR Hiroshimakinen Hospital, Kobe Century Memorial Hospital, Kobe University Hospital, Kochi Medical School Hospital, Koga General Hospital, Kumamoto Regional Medical Center, Kumamoto University Hospital, Kushiro Rosai Hospital, Kyorin University Hospital, Kyoto University Hospital, Maebashi Red Cross Hospital, Matsue City Hospital, Matsushita Memorial Hospital, Matsuyama Shimin Hospital, Minoh City Hospital, Mitoyo General Hospital, Miyagi Cancer Center, Mizushima Kyodo Hospital, Nagano Municipal Hospital, Nagasaki University Hospital, Nakagami Hospital, Nakano General Hospital, Nanpuh Hospital, National Cancer Center Hospital, National Cancer Center Hospital East, National Hospital Organization Fukuyama Medical Center, National Hospital Organization Kyushu Cancer Center, National Hospital Organization Oita Medical Center, National Hospital Organization Osaka Medical Center, National Hospital Organization Sendai Medical Center, National Hospital Organization Tokyo Medical Center, National Hospital Organization Yokohama Medical Center, Niigata Cancer Center Hospital, Niigata University Medical and Dental Hospital, Nikko Memorial Hospital, Nippon Medical School Hospital, Obihiro Tokushukai Hospital, Oita University Hospital, Okayama Saiseikai General Hospital, Okayama University Hospital, Onomichi Municipal Hospital, Osaka City General Hospital, Osaka Medical Center for Cancer and Cardiovascular Diseases, Osaka Medical College Hospital, Osaka Police Hospital, Osaka Red Cross Hospital, Saiseikai Chuwa Hospital, Saiseikai Kumamoto Hospital, Saiseikai Nakatsu Hospital, Saiseikai Niigata Daini Hospital, Saiseikai Noe Hospital, Sakai Municipal Mikuni Hospital, Saku Central Hospital, Seirei Hamamatsu General Hospital, Sendai Kousei Hospital, Shimane Prefectural Central Hospital, Shirakawa Clinic, Shizuoka General Hospital, Showa General Hospital, Suita Municipal Hospital, Sumitomo Hospital, Tenri Hospital, The University of Tokyo Hospital, Tochigi Cancer Center, Tohoku Rosai Hospital, Tokushima Red Cross Hospital, Tokyo Medical University, Tokyo Metropolitan Bokutoh Hospital, Tokyo Metropolitan Hiroo Hospital, Tokyo Metropolitan Police Hospital, Tokyo Women's Medical University (Institute of Gastroenterology), Tokyo Women's Medical University Hospital, Tonan Hospital, Toranomon Hospital, University Hospital Kyoto Prefectural University of Medicine, University of Fukui Hospital, University of the Ryukyus Hospital, University of Yamanashi Hospital, Wakayama Medical University Hospital,
Yao Municipal Hospital, Yokohama City University Medical Center, Yokohama Municipal Citizen's Hospital, and Yuai Memorial Hospital.

\section{References}

1. Maruyama K, Kaminishi M, Hayashi K, et al. Gastric cancer treated in 1991 in Japan: data analysis of nationwide registry. Gastric Cancer. 2006;9:51-66.

2. Isobe Y, Nashimoto A, Akazawa K, et al. Gastric cancer treatment in Japan: 2008 annual report of the JGCA nationwide registry. Gastric Cancer. 2011;4:301-16.

3. Nashimoto A, Akazawa K, Isobe Y, et al. Gastric cancer treated in 2002 in Japan: 2009 annual report of the JGCA nationwide registry. Gastric Cancer. 2013;1:1-27.

4. Ono H, Kondo H, Gotoda T, et al. Endoscopic mucosal resection for treatment of early gastric cancer. Gut. 2001;48:225-9.

5. Oda I, Gotoda T, Hamanaka H, et al. Endoscopic submucosal resection for early gastric cancer: technical feasibility, operation time and complications from a large consecutive series. Dig Endosc. 2005; 17:54-8.

6. Gotoda T, Yanagisawa A, Sasako M, et al. Incidence of lymph node metastasis from early gastric cancer: estimation with a large number of cases at two large centers. Gastric Cancer. 2000;3:219-25.

7. Tada M, Murakami A, Karita M, et al. Endoscopic resection of early gastric cancer. Endoscopy. 1993;25:445-50.

8. Tanabe S, Koizumi W, Mitomi H, et al. Clinical outcome of endoscopic aspiration mucosectomy for early gastric cancer. Gastrointest Endosc. 2002;56:708-13.

9. Park YM, Cho E, Kang HY, et al. The effectiveness and safety of endoscopic submucosal dissection compared with endoscopic mucosal resection for early gastric cancer: a systematic review and metaanalysis. Surg Endosc. 2011;25:2666-77.

10. Oda I, Saito D, Tada M, et al. A multicenter retrospective study of endoscopic resection for early gastric cancer. Gastric Cancer. 2006;9:262-70.

11. Watanabe K, Ogata S, Kawazoe S, et al. Clinical outcomes of EMR for gastric tumors: historical pilot evaluation between endoscopic submucosal dissection and conventional mucosal resection. Gastrointest Endosc. 2006;63:776-82.

12. Tanabe $\mathrm{S}$, Ishido $\mathrm{K}$, Higuchi $\mathrm{K}$, et al. Long-term outcomes of endoscopic submucosal dissection for early gastric cancer: a retrospective comparison with conventional endoscopic resection in a single center. Gastric Cancer. 2014;17:130-6.

13. Isomoto H, Shikuwa S, Yamaguchi N, et al. Endoscopic submucosal dissection for early gastric cancer. A large-scale feasibility study. Gut. 2009;58:331-6.

14. Goto O, Fujishiro M, Kodashima S, et al. Outcomes of endoscopic submucosal dissection for early gastric cancer with special reference to validation for curability criteria. Endoscopy. 2009;41:118-22.

15. Cho JH, Cha SW, Kim HG, et al. Long-term outcomes of endoscopic submucosal dissection for early gastric cancer: a comparison study to surgery using propensity score-matched analysis. Surg Endosc. 2016;30:3762-73.

16. Kim DY, Hong SJ, Cho GS, et al. Long-term efficacy of endoscopic submucosal dissection compared with surgery for early gastric cancer: a retrospective cohort study. Gut Liver. 2014;8:519-25.

17. Gotoda T, Iwasaki M, Kusano C, et al. Endoscopic resection of early gastric cancer treated by guideline and expanded National Cancer Center criteria. Br J Surg. 2010;97(6):868-71. 
18. Yamaguchi $\mathrm{N}$, Isomoto $\mathrm{H}$, Fukuda $\mathrm{E}$, et al. Clinical outcomes of endoscopic submucosal dissection for early gastric cancer by indication criteria. Digestion. 2009;80(3):173-81.

19. Tanabe S, Ishido K, Matsumoto T, et al. Long-term outcomes of submucosal dissection for early gastric cancer: a multicenter cllaborative study. Gastric Cancer. 2016. doi:10.1007/s10120016-0664-7.

20. Kurokawa $\mathrm{Y}$, Hasuike $\mathrm{N}$, Ono $\mathrm{H}$, et al. A phase II trial of endoscopic submucosal dissection for mucosal gastric cancer.: Japan Clinical Oncology Group study JCOG0607. Jpn J Clin Oncol. 2009;39(7):464-6.
21. Takizawa K, Takashima A, Kimura A, et al. A phase II clinical trial of endoscopic submucosal dissection for early gastric cancer of undifferentiated type: Japan Clinical Oncology Group study JCOG1009/1010. Jpn J Clin Oncol. 2013;43(1):87-91.

22. Oda I, Shimazu T, Ono H, et al. Design of Japanese multicenter prospective cohort study of endoscopic resection for early gastric cancer using Web registry (J-WEB/EGC). Gastric Cancer. 2012;15(4):451-4.

23. Kikuchi S, Katada N, Sakuramoto S, et al. Survival after surgical treatment of early gastric cancer: surgical techniques and longterm survival. Arch Surg. 2004;389:69-74. 